\title{
Medication Errors in Chemotherapy Preparation and Administration: a Survey Conducted among Oncology Nurses in Turkey
}

\author{
Arife Ulas ${ }^{1 *}$, Kamile Silay ${ }^{2}$, Sema Akinci ${ }^{3}$, Didem Sener Dede ${ }^{4}$, Muhammed \\ Bulent Akinci ${ }^{4}$, Mehmet Ali Nahit Sendur ${ }^{4}$, Erdem Cubukcu ${ }^{5}$, Hasan Senol \\ Coskun $^{6}$, Mustafa Degirmenci ${ }^{7}$, Gungor Utkan ${ }^{8}$, Nuriye Ozdemir ${ }^{4}$, Abdurrahman \\ Isıkdogan', Abdullah Buyukcelik ${ }^{10}$, Mevlude Inanc ${ }^{11}$, Ahmet Bilici ${ }^{12}$, Hatice \\ Odabasi $^{13}$, Sener Cihan ${ }^{14}$, Nilufer Avci ${ }^{15}$, Bulent Yalcin ${ }^{4}$
}

\begin{abstract}
Background: Medication errors in oncology may cause severe clinical problems due to low therapeutic indices and high toxicity of chemotherapeutic agents. We aimed to investigate unintentional medication errors and underlying factors during chemotherapy preparation and administration based on a systematic survey conducted to reflect oncology nurses experience. Materials and Methods: This study was conducted in 18 adult chemotherapy units with volunteer participation of 206 nurses. A survey developed by primary investigators and medication errors (MAEs) defined preventable errors during prescription of medication, ordering, preparation or administration. The survey consisted of 4 parts: demographic features of nurses; workload of chemotherapy units; errors and their estimated monthly number during chemotherapy preparation and administration; and evaluation of the possible factors responsible from ME. The survey was conducted by face to face interview and data analyses were performed with descriptive statistics. Chi-square or Fisher exact tests were used for a comparative analysis of categorical data. Results: Some $83.4 \%$ of the 210 nurses reported one or more than one error during chemotherapy preparation and administration. Prescribing or ordering wrong doses by physicians $(65.7 \%)$ and noncompliance with administration sequences during chemotherapy administration $(50.5 \%)$ were the most common errors. The most common estimated average monthly error was not following the administration sequence of the chemotherapeutic agents (4.1 times/month, range 1-20). The most important underlying reasons for medication errors were heavy workload $(49.7 \%)$ and insufficient number of staff $(36.5 \%)$. Conclusions: Our findings suggest that the probability of medication error is very high during chemotherapy preparation and administration, the most common involving prescribing and ordering errors. Further studies must address the strategies to minimize medication error in chemotherapy receiving patients, determine sufficient protective measures and establishing multistep control mechanisms.
\end{abstract}

Keywords: Medication errors - chemotherapy - nursing - chemotherapeutic drug preparation/administration

Asian Pac J Cancer Prev, 16 (5), 1699-1705

\section{Introduction}

The number of cancer patients is gradually increasing worldwide including our country. Therefore, the numbers of patients that are receiving chemotherapeutic agents are increasing. This upsurge results in unwanted mistakes during chemotherapy preparation and administration. Medication errors among the most common medical errors that are threatening patient safety. Oncologic medication errors might lead severe problems due to low

${ }^{1}$ Department of Medical Oncology, ${ }^{3}$ Department of Hematology, Ankara Ataturk Training and Research Hospital, ${ }^{2}$ Department of Internal Medicine and Geriatrics, ${ }^{4}$ Department of Medical Oncology, Faculty of Medicine, Yildirim Beyazit University, Ataturk Research and Training Hospital, Ankara, ${ }^{5}$ Department of Medical Oncology, Ali Osman Sonmez Oncology Hospital, Bursa, ${ }^{6}$ Department of Medical Oncology, Faculty of Medicine, Akdeniz University, Antalya, ${ }^{7}$ Department of Medical Oncology, Izmir Tepecik Training and Research Hospital, Izmir, ${ }^{8}$ Department of Medical Oncology, Ankara University School of Medicine, Ankara, ${ }^{9}$ Department of Medical Oncology, Faculty of Medicine, Dicle University, Diyarbakır, ${ }^{10}$ Department of Medical Oncology, Acıbadem

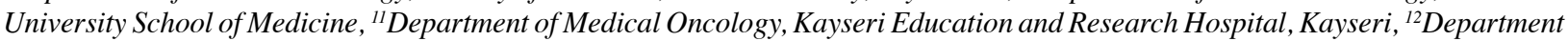
of Medical Oncology, Faculty of Medicine, Istanbul Medipol University, ${ }^{13}$ Department of Medical Oncology, Lutfi Kirdar Kartal Education and Research Hospital, ${ }^{14}$ Department of Medical Oncology, Istanbul Okmeydanı Training and Research Hospital, Istanbul, ${ }^{15}$ Department of Medical Oncology, Balıkesir Government Hospital, Balıkesir, Turkey*For correspondence: drarifeulas@hotmail.com 
therapeutic index and high toxicity of chemotherapeutic agents (Gaudio and Menonna, 1998). Chemotherapeutics are among the high-risk medications according to ISMP (Institute for Safe Medication Practices) guidelines (Institute for Safe Medication Practices, 2012).

Medication errors might be seen at any stage of the treatment process, from the prescribing medication to administration. Medication errors were accepted a significant health problem in 1999 and reported that 7.000 died, and 80.000 hospitalized every year due to these errors in United States (Kohn, Corrigan and Donaldson, 2000). Similar results also reported in the Canadian Study (Baker et al., 2004). A prospective study demonstrated that the rate of chemotherapeutic agent administration error was $0.04 \%$ (Ford et al., 2006). Dosing errors either low or high dose, wrong timing, administration error, wrong medication, infusion rate errors, wrong administration route, forgetting to give medication or hydration, giving the medication to wrong patient are chemotherapeutic agent administration errors (Dwight, 2002). The studies revealed that the most common error happens during chemotherapy ordering period (Leape et al., 1995; Gandhi et al., 2005).

The mortality, morbidity and increased cost caused development of national guidelines including multidisciplinary medication and prescription rules, preparation and distribution methods and medication management. Studies suggest that computer monitarization system, computerized prescriber order entry (CPOE) and prophylactic computer alarm methods might decrease medication administration errors (Classen et al., 1991; Markert et al., 2009; Kukreti et al., 2014).

Medication errors should be addressed in order to increase patient safety and also quality of cancer treatment. Initially prescription, transcription, dispensing and administration phases need to be corrected. In this period, the role of nurses is crucial. Right medication management (nine rights: right medication, right patient, right dose, right time, right administration, right medication , right registration- right form, right sequence, right administration duration) is essential. However, medication management became harder and complicated due to new treatment protocols and different administration techniques. Unfortunately reporting of these medication errors is insufficient (Wolf et al., 2000; Koohestani et al., 2010). Recognizing the main factors and underlying reasons that are contributing medication errors is very important to create a safe environment for patients.

We aim to define the medication errors and the underlying factors during chemotherapy preparation and administration based on oncology nurses experience in order to create a safe environment for patients.

\section{Materials and Methods}

\section{Research design}

This study is a multicenter cross-sectional study. The study conducted by volunteer participation of 206 adult oncology nurses in 18 Turkish oncology centers between June, 2014-August, 2014. The study approved by Ataturk Research and Training Hospital Ethics Committee. The study aims to define medication errors during prescription, ordering, preparation and administration phases based on oncology nurses experience, define the contributing factors and determine the frequency (and monthly number of these errors).

\section{Survey instrument}

The survey was created based on literature data (Ford et al., 2006; Koohestani HR et al., 2010). Medication preparation and administration errors (MAEs) was defined as the preventable errors that occurred during medication prescription, ordering and administrating (wrong medication, wrong dose, wrong administration, wrong time) period (Lassetter et al., 2003). The investigators agreed upon survey questions. Since this subject is a very sensitive, volunteer participation was required. Ethical rules are taken into account. The name of participants was kept confidential. Survey was administered in a safe environment. The inclusion criteria for participation was to be physically and mentally healthy, to work in the chemotherapy unit in the last six months and to be a volunteer. Survey was initially evaluated after the eight subjects participation in terms of intelligibility and clarity of questions. Investigators interviewed the subjects face to face.

The survey consists of four parts. In the first part the demographic and professional features of nurses (age, education level, institution, work experience (year), the period that she is working in the chemotherapy unit). Also, whether they have chemotherapy education certificate, sufficient knowledge about chemotherapy preparation and administration or not questioned. The second part consists of the questions that are evaluating workload of chemotherapy units ( weekly chemotherapy preparation number (single chemotherapy, multiple chemotherapy, elastic pump infusions), weekly chemotherapy administration number (single chemotherapy, multiple chemotherapy, elastic pump infusion). In the third part, prescribing and administrating errors of chemotherapeutics and their estimated monthly frequency were questioned ( prescribing or ordering wrong medication / wrong dosing, prescribing medication to wrong patient, placing wrong medication into chemotherapy solution, forgetting to place medication into solution, giving either lower or higher dose than ordered, wrong labeling, administrating medication to wrong patient, do not obey the administration time and/ or sequence).

In the last part, the most relevant and important factors that are contributing to medication errors during chemotherapy preparation and administration were questioned. Participants allowed to choose one or more than one option. Investigators interrogated the participants face to face. They explained the questions in detail in order to prevent misunderstandings. The reported errors were classified into two groups; either prescribing and ordering errors or prescribing and administrating errors.

The content validity of the questionnaire was evaluated by experts including five academic and five clinical nurses. Each expert has been asked to evaluate each item on the form. At the end of the evaluation, the experts did not recommend any changes to the questionnaire and 
Medication Errors in Chemotherapy Preparation and Administration: A Survey of Oncology Nurses in Turkey

approved the suitability and content validity of the items.

\section{Statistical analyses}

Data analyses were performed by descriptive statistics (table, mean, median). Chi-square or Fisher exact tests were used for a comparative analysis of categorical data. Statistical analyses were performed using SPSS 15.0 software (SPSS Inc. Chicago, IL, USA). All statistical assessments were two-sided, and a $\mathrm{p}$ value of $<0.05$ was considered as statistically significant.

\section{Results}

\section{The demographic features of nurses}

Total 206 nurses participated in the survey. The median age of nurses was 35 years (range 20-53), 69.4\% of them was a university graduate, $59.2 \%$ of them working as a nurse at least ten years or more. The number of nurses working in chemotherapy units 3 or more years was 110 . $36.9 \%$ of nurses has chemotherapy education certificate. $\%$ 59.2 of them reported that they had sufficient information about chemotherapy preparation and administration.

While $35.9 \%$ of them was preparing the chemotherapy more than 30 times per week, $55.8 \%$ of them administrating the chemotherapy more than 30 times per week. $47 \%$ of the nurses both prepare and administer the chemotherapeutics, and $44.7 \%$ of them was administered by themselves. While $30.1 \%$ of them was preparing single chemotherapy, $36.4 \%$ of them was administrating single chemotherapy more than 30 times per week. $38.8 \%$ of them was preparing multiple chemotherapies while $49.5 \%$ of them was administrating multiple chemotherapies more than 30 times per week. While $16.5 \%$ of them was preparing 48 hours elastic infusion pump chemotherapy, $15.2 \%$ of them was administrating multiple chemotherapies more than 30 times per week (Table 1).

\section{Types of error}

$171(83.4 \%)$ nurses reported that they had experienced at least one undesirable error while preparing or administrating chemotherapeutics during their professional life. Based on $65.7 \%$ of nurses experience the most common error was prescribing or ordering wrong doses by physicians. Not following the sequence of multi chemotherapy administration (50.5\%), prescribing or ordering wrong medication by physician $(43.1 \%)$ and not following the infusion time of multi chemotherapy administration $(39.7 \%)$ were the following error types. When we review the average error during one month period, for not following administration time, the average monthly error number was 4.1 times/month (1-20), for prescribing or ordering wrong dose by physician, 3 times/month (1-20) and for not following the sequence of chemotherapeutic agents, 3.2 times/month (1-20) (Table 2).

$69.2 \%$ of nurses reported that the administration of medication not completed in the required time with elastic infusion pump. 55\% of nurses reported that more than $5 \%$ of medication remained in the pump. $27 \%$ of nurses reported that patients came to outpatient chemotherapy unit with sufficient information. $30.9 \%$ claimed that the
Table 1. Nurses Characteristic

\begin{tabular}{|c|c|}
\hline Features (Total 206 nurses) & $\mathrm{N}(\%)$ \\
\hline \multicolumn{2}{|l|}{ Age (median: 35 years, range: $20-53$ ) } \\
\hline \multicolumn{2}{|l|}{ Education Level } \\
\hline Health Vocational High School & $34(16.5)$ \\
\hline Health Vocational College & $29(14.1)$ \\
\hline University & $143(69.4)$ \\
\hline \multicolumn{2}{|l|}{ Institution } \\
\hline Community Hospital & $34(16.5)$ \\
\hline Training and Research Hospital & $80(38.8)$ \\
\hline University & $73(35.4)$ \\
\hline Private Hospital & $19(9.2)$ \\
\hline \multicolumn{2}{|l|}{ Work duration(year) } \\
\hline $0-5$ & $37(18)$ \\
\hline $5-10$ & $47(22.8)$ \\
\hline 10 and more & $122(59.2)$ \\
\hline \multicolumn{2}{|l|}{ Work duration in CAU } \\
\hline Less than 1 year & $36(17.5)$ \\
\hline $1-3$ year & $60(29.1)$ \\
\hline 3 years and more & $110(53.4)$ \\
\hline \multicolumn{2}{|l|}{ CT Education Certificate } \\
\hline Present & $76(36.9)$ \\
\hline Absent & $130(63.1)$ \\
\hline \multicolumn{2}{|c|}{ Adequate education about $\mathrm{CT}$ preparation and administration } \\
\hline Yes & $122(59.2)$ \\
\hline No & $17(8.3)$ \\
\hline Partially & $67(32.5)$ \\
\hline \multicolumn{2}{|c|}{ Chemotherapy preparation number (weekly) } \\
\hline $1-10$ & $33(16.0)$ \\
\hline $11-30$ & $29(14.1)$ \\
\hline $30<$ & $74(35.9)$ \\
\hline None & $70(34.0)$ \\
\hline \multicolumn{2}{|c|}{ Chemotherapy administration number (weekly) } \\
\hline $1-10$ & $38(18.4)$ \\
\hline $11-30$ & $41(19.9)$ \\
\hline $30<$ & $115(55.8)$ \\
\hline None & $12(5.8)$ \\
\hline \multicolumn{2}{|c|}{ Both prepare and administration (\%, weekly) $(\mathrm{n}=97)$} \\
\hline \multicolumn{2}{|l|}{ Average: \% 44.7 (\%2-100) } \\
\hline \multicolumn{2}{|c|}{ Single chemotherapy preparation number (weekly) } \\
\hline $1-10$ & $47(22.8)$ \\
\hline $11-30$ & $34(16.5)$ \\
\hline $30<$ & $62(30.1)$ \\
\hline None & $63(30.6)$ \\
\hline \multicolumn{2}{|c|}{ Single chemotherapy administration number (weekly) } \\
\hline $1-10$ & $64(31.1)$ \\
\hline $11-30$ & $53(25.7)$ \\
\hline $30<$ & $75(36.4)$ \\
\hline None & $14(6.8)$ \\
\hline \multicolumn{2}{|c|}{ Multiple chemotherapy preparation numbers (weekly) } \\
\hline $1-10$ & $30(14.6)$ \\
\hline $11-30$ & $36(17.5)$ \\
\hline $30<$ & $80(38.8)$ \\
\hline None & $60(29.1)$ \\
\hline \multicolumn{2}{|c|}{ Multiple chemotherapy administration numbers (weekly ) } \\
\hline $1-10$ & $35(17.0)$ \\
\hline $11-30$ & $55(26.7)$ \\
\hline $30<$ & $102(49.5)$ \\
\hline None & $14(6.8)$ \\
\hline
\end{tabular}

48 hours elastic infusion pump chemotherapy preparation number
$1-10$
$36(17.5)$
$11-30$
$39(18.9)$
$30<$
$34(16.5)$
None
$97(47.1)$

48 hours elastic infusion pump chemotherapy administration number

$\begin{array}{ll}1-10 & 78(38.2) \\ 11-30 & 61(29.9) \\ 30< & 31(15.2) \\ \text { None } & 34(16.7)\end{array}$

*CT, Chemotherapy; CAU, Chemoterapy preparation and administration unit 
Table 2. Types of Medication Errors in Chemotherapy Preparation and Administration

\begin{tabular}{|c|c|c|}
\hline Error Types & $\mathrm{N}(\%)$ & $\begin{array}{l}\text { Estimated average monthly } \\
\text { error number*, mean (range) }\end{array}$ \\
\hline \multicolumn{3}{|l|}{ 1. Errors during chemotherapy prescribing and ordering } \\
\hline Prescribing or ordering wrong medication & $88(43.1)$ & $2.12(1-15)$ \\
\hline Prescribing or ordering wrong doses & $134(65.7)$ & $3.0(1-20)$ \\
\hline Prescribing or ordering to the wrong patient & $44(21.6)$ & $0.70(1-5)$ \\
\hline \multicolumn{3}{|l|}{ 2. Errors during chemotherapy preparation and administration } \\
\hline $\begin{array}{l}\text { Errors during labeling of chemotherapy serums } \\
\text { (patient name, medication name and dose, spelling errors etc.) }\end{array}$ & $71(34.8)$ & $2.19(1-10)$ \\
\hline Ordering errors (ordering less or more medication than required) & $68(33.3)$ & $1.31(1-15)$ \\
\hline Adding wrong medication into chemotherapy serum & $35(17.2)$ & $1.41(1-8)$ \\
\hline Forgetting adding medication into chemotherapy serum & $17(8.3)$ & $0.82(1-3)$ \\
\hline Administrating the chemotherapy to wrong patient & $43(21.1)$ & $0.53(1-3)$ \\
\hline Not following chemotherapy sequence during multichemotherapy administration & $103(50.5)$ & $3.2(1-20)$ \\
\hline Not following the administration duration & $81(39.7)$ & $4.1(1-20)$ \\
\hline \multicolumn{3}{|l|}{ Table 3. Contributing Factors to Medication Errors } \\
\hline \multicolumn{2}{|l|}{ Reasons } & $\mathrm{N}(\%)^{*}$ \\
\hline \multicolumn{2}{|l|}{ High work load } & $99(49.7)$ \\
\hline \multicolumn{2}{|l|}{ Insufficient staff } & $72(36.5)$ \\
\hline \multicolumn{2}{|l|}{ Inadequate education/experience about chemotherapy } & $35(19.9)$ \\
\hline \multicolumn{2}{|l|}{ Inadequate continuous education about chemotherapy } & $37(20.7)$ \\
\hline \multicolumn{2}{|l|}{ Inadequate communication during sign outs( lunch break etc) } & $20(11.8)$ \\
\hline \multicolumn{2}{|l|}{ Protocol writing errors (ineligible doctor writing) } & $30(16.7)$ \\
\hline \multicolumn{3}{|c|}{ Insufficient physical conditions (Crowded working environment, telephone, space-constrained, noise, having patients' relatives } \\
\hline \multicolumn{2}{|c|}{ around during chemotherapy etc.) } & $26(14.1)$ \\
\hline \multicolumn{2}{|l|}{ Stress, tiredness and burn out syndrome } & $50(25.6)$ \\
\hline \multicolumn{2}{|l|}{ Inadequate information about patient who is receiving chemotherapy } & $21(12.7)$ \\
\hline \multicolumn{2}{|l|}{ Neglecting of chemotherapy units by hospital or department administrators } & $33(17.8)$ \\
\hline
\end{tabular}

* One or more option stated

required information has not been given to patients at all, and $42.2 \%$ reported that the given information was insufficient.

\section{Contributing factors to medication errors}

The underlying reasons of medication errors was reported as (more than one option was given) increased workload (49.7\%), inadequate number of staff (36.5\%) and burnout syndrome (25.6\%) (Table 3).

Significant difference found between university graduates and nonuniversity graduates when the association between the features of nurses and error types evaluated. The rate of recognition of prescribing and ordering errors was shown higher among university graduates $(\mathrm{P}=0.001)$. When nurses with long working years compared with less working years, the prescribing and ordering errors were more common $(p=0.044)$. The probability of recognizing infusion pump errors were higher among the nurses who work in chemotherapy units more than three years (59\%), and the difference between the groups was significant $(p=0.029)$. When nurses who prepare chemotherapy more than 30 times weekly compared with the less than 30 , the rate of physician prescribing and ordering errors has been shown higher. There was a significant difference between the groups $(\mathrm{p}<0.001)$. No significant association was shown between the features of nurses and the other error types.

\section{Discussion}

Medication errors of chemotherapeutic agents is a serious problem leading to increasing in morbidity, mortality, hospital staying length and medical expenses. In our knowledge, this is the first study reporting undesired errors during chemotherapy preparation and administration. In this study we interrogated whether we practice nine right in medication administration (right medication, right patient, right dose, right time, right administration, right medication, right registrationright form, right sequence, right administration time) adequately or not. Our results are remarkably interesting and engrossing.

There are different methods for measuring medical errors. Self-reporting and nonself-reporting are among those methods. The frequency of reporting medical errors is depending on the methodology, patient population and also a thoroughness of defining these errors. We preferred the nurses' perspective in this study for the couple of reasons. First of all, the probability of nurse's recognition of medical error is high since they are the primary administrator of medications. Nurses play an important role during the process of preparation and administration, and they compare the prescription with the medication that comes from a pharmacy. The third one the medical errors can be prevented with increasing the quality of nursing. As we mentioned before nurses are responsible of medication administration (O'Shea, 1999; Burke, 2005; Catalano, 2005).

In this survey, $83.4 \%$ of nurses reported at least one chemotherapy preparation and administration error which is done either by themselves or somebody else during their professional life. We have detected higher error rates 
than previous studies. In previous two survey studies the medication error rates were reported as $42.1 \%$ and 43 $\%$ (Lisby et al., 2005; Mrayyon et al., 2007). In another survey reporting errors from nurse's perspective showed that the error rate was $64.5 \%$ (Cheragi et al., 2013). A survey study that was done in our country showed that two third of nurses reported that they had made one or more than one error during their career (Guneş et al., 2014).

The most common mistake that was shown in our study was prescribing or ordering wrong dose of medication. According to many studies medication errors, usually occur during prescription or administration phases and accounted for $65 \%$ to $87 \%$ of all medication errors (Bates et al., 1995; Benjamin, 2003). A retrospective study showed that errors most commonly occurs during chemotherapy prescribing and ordering phase and $45 \%$ of them were preventable (Gandhi et al., 2005). Another study revealed that $39 \%$ of errors occur in chemotherapy ordering phase; $28 \%$ of them occur while calculating the medication dose (Leape et al.,1995). Administration of the wrong dose accounted for $39 \%$ of the 140 errors reported by chemotherapy nurses (Schulmeister, 1999). The study that is evaluating the chemotherapy prescribing errors (the most common error) was detected 135 errors in 43.188 parenteral cytotoxic agent (3.1/1000 chemotherapy) preparation. The error types were administrating the wrong dose or forgetting to administer the medication at all, wrong medication, wrong frequency error, wrong treatment period, wrong patient, wrong administration rate and wrong administration route (Carrasco et al., 2007).

The second most common mistake in our study was not following the administration sequence in multi chemotherapy protocols. The following mistakes were prescribing and ordering wrong medication and not following the ordered infusion time. The rate of these errors was higher than previously reported ones (Han et al., 2005; Young et al., 2010). The study that was including outpatient cancer patients showed that medication administration errors were done in $7 \%$ of 262 adult patients (Walsh et al., 2009). Forgetting to administer the medication is also among common medication errors (Anselmi, Peduzzi and Santos, 2007). In our study forgetting to place medication into the serum rate was 8.3\%. Wrong time (\%36), wrong method (\%19) and wrong dose $(\% 15)$ was reported in another observational study including 336 patients (Port et al., 2005). When we compare our study with this one wrong time rate $(39.7 \%)$ was found similar but the other error rates were found higher.

One of the main underlying factors of unwanted medication errors has been found as the low ratio of nurse to patient (Stratton et al., 2004). From nurses perspective the factors that were leading medication errors were tiredness due to crowded and noisy environment, inadequate support system, carelessness and high work load (Schulmeister, 1999; Haw et al., 2005). According to our findings majority of nurses believed that these errors were multifactorial. Consistent with previous studies, the most frequent two reasons of errors are high work volume and inadequate number of staff (Osborne et al., 1999; Beyea et al., 2003; Park et al., 2011). Units with the higher number of chemotherapy administration rates reported higher frequency of prescription and ordered errors. Also, one may consider stressful, and high pressure was working environment, high workload and limited time, the high number of rotation and turnover of physicians as other factors of prescription and ordering errors (Worthington, 2001; Beyea et al., 2003; Tang et al., 2007).

The errors of sequence and duration of chemotherapeutic agents suggest that nurses have inadequate education. One of the remarkable points of our study was that $63.1 \%$ of nurses did not have chemotherapy education certificate. The education of nurses is not standard, and they do not have enough information about medications. On the other hand, nursing educations, programs do not provide enough information on biology and pharmacology to prescribe medications (Latter et al., 2001). The chemotherapy nurses admitted that the main reason of medication errors is a lack of experience (Schulmeister et al., 1999). Nowadays increasing number of developing new medication and complex chemotherapy protocols requires the need of continuously updated education.

$14.1 \%$ of nurses reported that they were interrupted while preparing chemotherapy by environmental factors (telephone, patients' relatives, and the closeness of outpatient and chemotherapy service). Nurses considered interruption during preparation and administration of medication as a primary reason for medication errors (Mayo and Duncan, 2004; Ulanimo et al., 2007). Therefore, nursing managers should provide enough nursing staff and a proper environment for nurses during preparation and administration of medication to prevent medication errors.

In this survey, the medication errors that identified have not been reported routinely. A nurse may forget to report an error, or may be unwilling to report or might be inadequate to identify. The problem is involuntary reporting. Since reporting may end up with finding someone guilty or has a context of blaming someone, it is usually underreported. Traditionally, nurses blame themselves for making an error when hospital systems should take the responsibility for failing to establish a safe working environment (Beyea, Hicks and Becker, 2003). Based on the recent surveys done recently at the major hospitals in USA, only less than half of the actual medication administration errors have been claimed to be reported (Osborne et al., 1999; Stratton et al., 2004). Based on a previous study, medication administration errors may be underreported due to low nurse to patient ratio, busy working environment and inexperienced staff (Ford et al., 2005). Our survey supports this conclusion. Previous studies have communicated that fear of risk of prestige loss and to protect themselves from patient reaction underreporting practice have been done intentionally for MAEs (Wakefield et al., 1999; Stratton et al., 2004). Proper and accurate reporting, consistent periodical analysis and protection of reported data is crucial for developing preventative medication administration error systems (Ferner and Coleman, 2005).

Comprehensive and effective monitarization systems which are preventing or at least minimizing the number of frequencies is essential. Multidisciplinary approach 
should be the integral part of detecting and preventing process of medical errors. It is recommended to establish a system focusing on the collection of accurate data with the computer system by pharmacists, education of nurses, improving working conditions, developing the policies of distribution and administration (O'Shea, 1999). The pharmacist may prevent the error by evaluating if the order entry is accurate. In a previous decade, it was reported that the most common medication error was ordering mistakes by physician (Bates et al., 1995; Leape et al., 1995). Despite all preventive measures chemotherapy ordering error was reported at least 4\% (Gandhi et al., 2005; Weis et al., 2005). All chemotherapy protocols were controlled in order to detect medication errors before they reach the patient with two different error followup system. This systems aim was to provide collaboration among the physician, nurse and pharmacist who monitarize chemotherapy errors. Electronic prescribing and ordering system decreased the chemotherapy errors to $4 \%$ in 2005 and $2.8 \%$ in 2006 . It was documented that this system decreased chemotherapy errors significantly and prevented them by $99.9 \%$ (Markert et al., 2009).

Computerized prescriber order entry systemic (CPOE) is an important and longed for technology that decreases medication errors. By using this system chemotherapy security increased and chemotherapy prescribing errors decreased (Jeon et al., 2014). The review that is investigating the effect of CPOE on medication errors showed that it decreased chemotherapy related medication errors in \% studies (Kukreti et al., 2014). When manual and computerized chemotherapy prescriptions compared; the error rate was found $20.4 \%$ in manual orders and $11.8 \%$ in computerized orders and the relative risk (RR) was decreased by $42 \%$ (Small et al., 2008).

Independent control procedures are effective in detecting errors (Ford et al., 2006). In many hospitals, the ordering, distribution and administration of medication should be controlled by independent pharmacist and nurse. The aim of this method is not controlling physician but to increase the safety of chemotherapy administration and productivity. Since the number of patients especially elderly patients with comorbidities that are receiving chemotherapy and newly developed complex CT protocols are increasing, these control system are mandatory. This optimal error detecting system should be trustworthy, cheap, easily accessible and can be easily used in the majority of hospitals.

Patient safety, decreasing the incidence of chemotherapeutic errors, application of specific strategies are the primary goals of a successful treatment. For this reason independent double-check (IDC) system was established providing patient safety during administration of high risk medications. Nurses must learn and apply the standard practicing guidelines especially IDC for increasing patient safety (Baldwin and Walsh, 2014). The principle rules of medication safety system to minimize the chemotherapy administration errors was specified by Gilbar and Dooley (Gilbar and Dooley, 2007). The role of human factor is extremely important in order to provide patient safety (Norris, 2009).

Our study has some limitations. The limitations of our study are; relatively small sample size, our data based on surveys and the patient charts or orders wee not controlled retrospectively, the errors were reported based on only nurses experience and providing pharmacist and physician based errors. The studies in the literature reported the errors based on patient records. In our country, it is impossible to control the records about medication errors due to insufficient medication monitarization, archiving system and medication error reporting system. On the other hand, the study is multicenter including both university and community hospitals and this is the strength of our study.

Obviously, there are challenges in avoiding the medication errors completely. Our priority should be minimize these errors. In this study, extremely important clinical problems such as a high work load in chemotherapy units and the inadequate chemotherapy administration education of nurses were addressed. The importance of integrating chemotherapeutic medication management into continuous nursing education. The staff should be encouraged to report medication errors and record them correctly. For this reason, hospital administrator should show a positive attitude regarding the reporting of medication error. Every hospital should improve the systems to detect and prevent the common medical errors while ordering, distribution and administration process.

In conclusion, our findings suggest that the medical errors are very common during chemotherapy preparation and administration and the most common ones are prescribing and ordering errors. We have faced with the reality of higher than expected medication error rates due to underreporting. Since the number and type of chemotherapeutic agents are increasing, patient safety in oncology is becoming more critical. The results of our survey clearly demonstrates the importance developing preventative strategies for Medication preparation and administration. In future large and credible studies this factor needs to be taken into consideration carefully by taking adequate measures to prevent medication errors by developing and implementing a multidisciplinary control mechanism. .

\section{Acknowledgements}

We would like to thank to our nursing staff for their kind collaboration. We acknowledge the following physicians for their contributions by assisting data collection (Yıldız R, Aksoy S, Turkoz FP, Esbah O, Sencan $\mathrm{O}$ and Ozal FG)

\section{References}

Anselmi M, Peduzzi M, Santos CI (2007). Errors in the administration of intravenous medication in Brazil hospitals. J Clin Nurs, 16, 1839-47.

Baker GR, Norton PG, Flintoft V, et al (2004). The Canadian Adverse Events Study: the incidence of adverse events among hospital patients in Canada. CMAJ, 170, 1678-86.

Baldwin K, Walsh V (2014). Independent double-checks for high-alert medications: essential practice. Nursing, 44, 65-7.

Bates DW, Cullen DJ, Laird N et al (1995). Incidence of adverse drug events and potential adverse drug events: implications 
Medication Errors in Chemotherapy Preparation and Administration: A Survey of Oncology Nurses in Turkey

for prevention. J Am Med Association, 274, 29-34.

Benjamin DM (2003). Reducing medication errors and increasing patient safety: case studies in clinical pharmacology. J Clin Pharmacol, 43, 768-83.

Beyea SC, Hicks RW \& Becker SC (2003). Medical errors in the OR-a secondary analysis of Medmarx. AORN Journal, 77, 132-4.

Burke KG (2005). The state of the science on safe medication administration symposium. J Infus Nurs, 28, 87-92.

Carrasco D, Pareja A, Yachachi A, Cortes F, Espuny A (2007). Prescription errors in chemotherapy. Farm Hosp, 313, 161- 4.

Catalano K (2005). Update on the national patient safety goals: Changes for 2005. AORN J, 81, 336-46.

Cheragi MA, Manoocheri H, Mohammed SR et al (2013).Types and causes of medication errors from nurse's viewpoint. Iran J Nurs Midwifery Res, 18, 3, 228-31.

Classen DC, Pestotnik SL, Evans RS, et al (1991). Computerized surveillance of adverse drug events in hospital patients. JAMA, 266, 2847-51,

Dwight DK (2002) Prevention of chemotherapy medication errors. J Pharmacy Practice, 15, 17-31.

Ferner RE, Coleman J (2005). Anticipating, preventing and investigating medication errors. Clin Med, 5, 12-15.

Ford CD, Killebrew J, Fugitt Pet al (2006). Study of medication errors on a community hospital oncology ward. J Oncol Pract, 2, 149-54.

Gandhi TK, Bartel SB, Shulman LN, et al (2005). Medication safety in the ambulatory chemotherapy setting. Cancer, 104, 2477-83.

Gaudio D, Menonna-Quinn D Chemotherapy (1998). Potential occupational hazards. Am J Nurs, 98, 59-65.

Gilbar PJ, Dooley MJ (2007). Review of case reports of inadvertent intrathecal administration of vincristine: Recommendations to reduce occurrence. Asia-Pac J Clin Oncol, 3, 59-65.

Guneş UY, Gurlek O, Sonmez M (2014). Factors contributing to medication errors in Turkey: nurses perspectives. J Nurs Manag, 22, 295-303.

Han PY, Coombes ID, Green B (2005). Factors predicative of intravenous fluid administration errors in Australian surgical care wards. Quality Safety Health Care, 14, 179-84.

Haw CM, Dickens G, Stubbs J (2005). A review of medication administration errors reported in a large psychiatric hospital in the United Kingdom. Psychiatr Serv, 56, 1610-3.

Institute for Safe Medication Practices (2012). ISMP's list of high-alert medications. http://www. ismp.org/tools/ highalertmedications.pdf.

Jeon J, Taneva S, Kukreti V, et al (2014). Toward successful migration to computerized physician order entry for chemotherapy. Curr Oncol, 21, 221-8.

Kohn LT, Corrigan JM, Donaldson MS (eds) (2000). To Err is human: building a safer health system. Washington, DC, National Academy Press, 1-24, 17-25

Koohestani HR, Baghcheghi N (2010). Barriers to the reporting of medication adminisraion errors an nursing students. Aust $J$ Adv Nurs, 27, 66-74.

Kukreti V, Cosby R, Cheung A et al (2014). Computerized prescriber order entry in the outpatient oncology setting: from evidence to meaningful use. Curr Oncol, 21, 604-12.

Lassetter JH, Warnick ML: Medical errors, drug-related problems, and medication errors (2003). J Nurs Care Qual, 18, 175-181.

Latter S, Rycroft-Malone J, Yerrell P, et al (2001). Nurses' educational preparation for a medication education role: findings from a national survey. Nurse Education Today, 21, 143-54.

Leape LL, Bates DW, Cullen DJ, et al (1995). Systems analysis of adverse drug events. ADE Prevention Study Group. JAMA, 274, 35-43.

Lisby M, Nielsen LP, Mainz J (2005). Errors in the medication process: frequency, type, and potential clinical consequences. Int J Qual Health Care, 17, 15-22.

Markert A, Thierry V, Kleber M, et al (2009). Chemotherapy safety and severe adverse events in cancer patients: strategies to efficiently avoid chemotherapy errors in in- and outpatient treatment. Int J Cancer, 124, 722-8.

Mayo AM, Duncan D (2004). Nurse perceptions of medication errors. What we need to know for patient safety. J Nurs Quality Care, 19, 209-17.

Mrayyon MT, Shishani K, Al-faouri L (2007). Rate, causes and reporting of medication errors in Jordan: Nurses' perspectives. J Nurs Manag, 15, 659-70.

Norris B (2009). Human factors and safe patient care. J Nurs Manag, 17, 203-11.

O'Shea E (1999). Factors contributing to medication errors: A literature review. J Clin Nurs, 8, 496-504.

Osborne J, Blais K, Hayes JS (1999). Nurses' perceptions when is it a medication error? J Nurs Adm, 29, 33-38.

Park SA, Lee SJ, Choi GU ( 2011). Survey of factors associated with nurses' perception of patient safety. Asian Pac J Cancer Prev, 12, 2129-32.

Port S, Fanton JE, Albertic C (2005). Drug administration errors and their determinants in pediatric inpatients. Int J Qual Health Care, 175, 381-9.

Small MDC, Barrett A, Price GM (2008). The impact of computerized prescribing on error rate in a department of oncology/ hematology. J Oncol Pharm Pract, 14, 181-87.

Schulmeister L (1999). Chemotherapy medication errors: descriptions, severity and contributing factors. Oncology Nursing Forum, 26, 1033-1042.

Stratton KM, Blegen MA, Pepper G et al (2004). Reporting of medication errors by pediatric nurses. J Pediatr Nurs, 19, 385-92.

Tang FI, Sheu SJ, Yu S, et al (2007). Nurses relate the contributing factors involved in medication errors. J Clin Nurs, 16, 447-57.

Ulanimo VM, Kelley CO, Connolly PM (2007). Nurses' perceptions of causes of medication errors and barriers to reporting. J Nursing Care Quality, 16, 1839-47.

Walsh KE, Dodd KS, Seetharaman K, et al (2009). Medication errors among adults and children with cancer in the outpatient setting. J Clin Oncol, 27, 891-6.

Wakefield DS, Wakefield BJ, Uden-Holman T, et al (1999). Understanding why medication administration errors may not be reported. Am J Med Qual, 14, 81-88.

Weis A, Haas P, Bauchm€uller K, et al (2005). "Severe adverse event" (SAE) documentation and analysis: a modern feature of good clinical practice (GCP) in cancer treatment. Onkologie, 212, 655.

Wolf ZR, Serembus JF, Smetzer J, et al (2000). Responses and concerns of healthcare providers to medication errors. Clinical Nurse Specialist, 14, 278-7.

Worthington K (2001) The health risks of mandatory overtime. Am J Nurs, 101, 96.

Young J, Slebodnik M, Sands L (2010). Bar code technology and medication administration error. J Patient Safety, 6, 115-20. 